\title{
Evaluation of Reagent Strips for Ascitic Fluid Leukocyte Determination: Is It a Possible Alternative for Spontaneous Bacterial Peritonitis Rapid Diagnosis?
}

\author{
Tarsila C.R. Ribeiroㅁ, Mario Kondo ${ }^{1}$, Ana Cristina C. Amaral ${ }^{1}$, Edson Roberto Parise ${ }^{1}$, \\ Maurício A. Bragagnolo Júnior ${ }^{1}$, Aécio Flávio Meirelles de Souza ${ }^{2}$ \\ ${ }^{1}$ Federal University of São Paulo - São Paulo Medical School; São Paulo, SP; ${ }^{2}$ Federal University of Juiz de Fora - HU; Juiz de Fora, MG, Brazil
}

\begin{abstract}
In order to evaluate the accuracy of a urine reagent dipstick (Multistix 10SG®) to determine ascitic fluid leukocyte count, we prospectively studied 106 cirrhotic patients from April 2003 to December 2004, in two different centers (Federal University of São Paulo - UNIFESP-EPM and Federal University of Juiz de Fora - HU-UFJF) for the rapid bedside diagnosis of spontaneous bacterial peritonitis. The mean age $54 \pm 12$ years, there was a predominance of males (eighty-two patients, $77 \%$ ), and alcohol was the most frequent etiology (43\%). Forty-four percent of patients were classified as Child $B$ and fifty-one as Child C $\mathbf{( 5 1 \% )}$. Abdominal paracentesis was performed both in outpatient and inpatient settings and the Multistix 10SG® was tested. Eleven cases of spontaneous bacterial peritonitis were identified by means of polymorphonuclear count. If we considered the positive Multistix 10SG® result of 3 or more, the sensitivity, specificity, positive and negative predictive value were respectively $71 \%, 99 \%, 91 \%$ and $98 \%$. With a positive reagent strip result taken as grade 2 (traces) or more, sensitivity was $86 \%$ and specificity was $96 \%$ with positive and negative predictive values of $60 \%$ and $99 \%$, respectively. Diagnostic accuracy was $95 \%$. We concluded that the use of a urine reagent dipstick (Multistix 10SG ${ }^{\circledR}$ ) could be considered a quick, easy and cheap method for ascitic fluid cellularity determination in SBP diagnosis.
\end{abstract}

Key-Words: Ascitic fluid, rapid diagnosis, dipstick, bacterial peritonitis.

Under no circumstances is spontaneous bacterial peritonitis (SBP) one of the most frequent and important complications found in cirrhotic patients with ascites, occurring in $10 \%$ to $30 \%$ of unselected patients admitted to hospital [1-5]. In-hospital mortality rate can reach $30 \%$ in spite of infection control measures, mortality being generally due to complications such as acute variceal bleeding, development of the hepatic-renal syndrome or progressive liver failure [2,3,5-8].

Although the combination of an elevated polymorphonuclear neutrophilic leukocyte (PMN) count and the yield of cultures of the ascitic fluid are considered the gold standard for the diagnosis of SBP, it has some shortcomings. First, the results of ascitic fluid culture are not readily available, postponing the diagnosis and treatment of the infection. Second, one of the most frequent variants of ascitic fluid infection is culture-negative neutrocytic ascites, which occurs in approximately $30 \%$ to $50 \%$ of patients $[4,10,11]$. Not only does the polymorphonuclear count seem to be accurate enough to determine which patients need antibiotic therapy but it has also been considered the easiest way to establish the diagnosis of SBP [12].

Although the polymorphonuclear count is faster than culture to make the diagnosis of SBP and reaches a good sensibility [13-15], it is promptly not available in every setting

Received on 19 July 2006; revised 18 December 2006.

Address for correspondence: Dr. Tarsila C.R. Ribeiro. Rua Doutor João Penido Filho, 327 - Bom Pastor - Juiz de Fora - MG. Zip code: 36021600. Telephone: (32)32118503 / 3212-3009 (fax) / (32) 9982-7499. E-mail: tarsila@acessa.com.

The Brazilian Journal of Infectious Diseases 2007;11(1):70-74. (C) 2007 by The Brazilian Journal of Infectious Diseases and Contexto Publishing. All rights reserved. (e.g. small cities); hence other ways of performing this diagnosis are under assessment. Reagent strips, traditionally used for prompt diagnosis of urinary tract infections, are now being applied to the diagnosis of other biological fluid infections such as meningitis, pleural empyema and spontaneous bacterial empyema [16-18]. This prospective study was undertaken to evaluate the use of a reagent strip in the diagnosis of ascitic fluid infection.

\section{Materials and Methods}

We prospectively studied 106 patients with cirrhotic ascites from two different University Centers (São Paulo Hospital/ UNIFESP- EPM and Universitary Hospital of Juiz de Fora/ HU-UFJF), from April 2003 to December 2004. The demographic characteristics of the study population are summarized in Table 1.

Table 1. Characteristics of 106 cirrhotic patients who underwent 200 paracentesis

\begin{tabular}{lc}
\hline Characteristics & Mean \pm SD or number (\%) \\
\hline Male sex & $82(77)$ \\
Age (years) & $54 \pm 12$ \\
Child-Pugh score & $10(5)$ \\
$\quad$ A & $89(44)$ \\
B & $101(51)$ \\
C & $17 \pm 8$ \\
MELD & \\
Etiology of cirrhosis & $46(43)$ \\
Alcohol abuse & $24(23)$ \\
Viral hepatitis & $13(12)$ \\
Alcohol + viral & $23(22)$ \\
Others &
\end{tabular}

* SD: standard deviation. 
The diagnosis of cirrhosis was based on clinical, biochemical and ultrasonographic findings [19], and patients underwent paracentesis both in outpatient and inpatient settings as dictated by standard medical practice. Patients with previous history of a surgical procedure in the previous four weeks were excluded from the study, as well as those with other causes of PMN increase in the ascitic fluid such as tuberculosis, pancreatitis and peritoneal carcinomatosis. The ethics committee from both centers approved the study.

First, peripheral blood samples were obtained for white blood cell (WBC), PMN counts, biochemical markers (glucose, albumin, creatinin, lactic dehydrogenase) and coagulation parameters evaluation.

At the same time, the patients underwent paracentesis and immediately after the procedure, an amount of ascitic fluid was sent to the laboratory in tubes containing heparin for determination of total and differential white blood cell counts. Another tube without anticoagulant was used for determination of protein, albumin, glucose and lactic dehydrogenase.

The cultures were seeded at bedside with the inoculation of $10 \mathrm{~mL}$ of ascitic fluid into blood culture bottles according to the study center. The ascitic fluid collected of patients from UNIFESP-EPM was inoculated on $25 \mathrm{~mL}$ of liquid blood culture (BD; BACTEC; Becton Dickinson and Company, County Clare, Ireland), and those from HU-UFJF on brain-heart infusion broth.

The reagent strip (Multistix 10SG®, Bayer Diagnostics) was immersed in $5 \mathrm{~mL}$ of ascitic fluid placed on a dry and clean container as described by the manufacturer for identification of leukocyte esterase. After two minutes, the reagent strip was read comparing the colour of the leukocyte reagent strip area with the colorimetric 5-grade scale depicted on the bottle. Based on the degree of colour change in the reagent strip area, the results were scored as negative, grade 1 or traces, grade 2 or low, grade 3 or moderate, and grade 4 or high. The test is based on the esterase activity of granulocytes present in the ascitic fluid that reacts with an ester releasing 3-Hydroxy5-phenyl-pyrrole.This reaction causes a color change in an azo dye (purple).

SBP was diagnosed when the PMN count was greater than $250 \mathrm{PMN} / \mathrm{mm}^{3}$ and the culture was positive, in the absence of another intra-abdominal source of infection. Culturenegative neutrocytic ascites was present when PMN was greater than $250 \mathrm{PMN} / \mathrm{mm}^{3}$ but the culture was negative, and monomicrobial bacterascites was defined as the presence of a single organism identified by the culture without an increased PMN count [4].

\section{Statistical Analysis}

Quantitative variables are expressed as mean \pm standard deviation and qualitative variables as percentage. Sensitivity, specificity, positive predictive value, negative predictive value, and accuracy were calculated. Positive and negative likelihood rates were also estimated.
A ROC curve was elaborated to determine the best positive cut-off point for the reagent strip.

\section{Results}

We analyzed the results of 200 samples of ascitic fluid collected from 106 cirrhotic patients. Most samples were collected in São Paulo Hospital (83\%) and in an outpatient setting (76\%). On sampling, eighteen patients $(9,5 \%)$ were on antibiotics for SBP prophylaxis.

In 29 cases, we had suggestive symptoms of SPB and the most frequent one was new onset encephalopathy (52\%), followed by abdominal pain (24\%) and fever (21\%). The diagnosis of SBP was established in 14 samples of ascitic fluid and culture was positive in $28 \%$ of cases (Table 2). The positivity of cultures was not influenced by differences in the blood culture bottles used ( $p>0.05$ ). Monomicrobial nonneutrocytic bacterioascites was observed in 8 (36\%) patients (Table 3).

The results of the reagent strip and the diagnosis of the 200 samples based on the PMN count were shown in Table 4. The correlation between ascitic fluid PMN count and the reagent strip results can be observed in Figure 1.

We also calculated the sensitivity, specificity, positive predictive value (PPV), negative predictive value (NPV), positive and negative likelihood ratio and accuracy of the reagent strip at different positive cut-off points ( $\geq 1, \geq 2$ and 23) (Table 5).

A ROC curve was elaborated to define which cut-off point was more reliable to be used as a positive result of the reagent strip for the diagnosis of SBP, by considering the accuracy of the test (Figure 2). The area under the curve and coordinates of the ROC curve are shown in Tables 6 and 7, respectively.

\section{Discussion}

This prospective study shows that ascitic fluid analysis by reagent strips has a reasonable sensitivity and specificity for SBP diagnosis. Different cut-off points were studied. We considered a reagent strip positive when the colorimetric scale was more than 2 (low) and a sensitivity of $71 \%$ and a specificity of $99 \%$ were achieved. The PPV and NPV were both very high in this setting (91\% and 98\%). On the other hand, reagent strip results more than 1 (traces) have an accuracy of 95\% with higher sensitivity (86\%). Although we lost a little in specificity (96\%) the NPV was still high (96\%). Actually, the low PPV (60\%) could be explained by the great percentage of ascitic fluid collected in the ambulatory setting and the low incidence of SBP in this population [20].

Considering the very high mortality of SBP the best cutoff point should be chosen based on the highest sensitivity achieved and the lowest false negative rate observed. This was reached with a cut-off point of 1 or more and was later confirmed by the results of the ROC curve.

The reagent strip test is based on the esterase activity of granulocytes and only activated PMN can release leukocyte esterase into the extracelullar milieu. Although the culture of 
Table 2. Multistix test, polymorphonuclear (PMN) count and culture of ascitic fluid in patients with spontaneous bacterial peritonitis (SBP)

\begin{tabular}{ccll}
\hline Patient & Multistix & $\mathbf{P M N} / \mathbf{m m}^{3}$ & Culture \\
\hline 1 & 2 & 428 & Negative \\
2 & 1 & 575 & Negative \\
3 & 3 & 896 & Negative \\
4 & 0 & 1,133 & Candida sp. \\
5 & 3 & 258 & Negative \\
6 & 3 & 312 & Escherichia coli \\
7 & 0 & 381 & Streptococcus sp. \\
8 & 1 & 419 & Negative \\
9 & 3 & 919 & Negative \\
10 & 4 & 3,098 & Negative \\
11 & 3 & 4,176 & Negative \\
12 & 4 & 4,464 & Negative \\
13 & 5 & 18,923 & Negative \\
14 & 5 & 54,000 & Streptococcus sp. \\
\hline
\end{tabular}

Table 3. Ascitic fluid bacteria isolated in patients with monomicrobial bacterascites

\begin{tabular}{lc}
\hline Bacteria isolated & Number (\%) \\
\hline Escherichia coli & $1(12.5)$ \\
Streptococcus sp. & $3(37.5)$ \\
Staphylococcus sp. & $4(50 \%)$ \\
\hline
\end{tabular}

Table 4. Final diagnosis of ascitic fluid samples and reagent strip test results

\begin{tabular}{lcccccc}
\hline \multirow{2}{*}{ Diagnosis } & \multicolumn{7}{c}{ Reagent strip results } \\
\cline { 2 - 7 } & N & $\mathbf{0}$ & $\mathbf{1}$ & $\mathbf{2}$ & $\mathbf{3}$ & $\mathbf{4}$ \\
\hline SBP & 4 & 2 & - & - & 1 & 1 \\
CNNA $^{*}$ & 10 & - & 2 & 1 & 5 & 2 \\
$\begin{array}{l}\text { Monomicrobial } \\
\text { bacterascites }\end{array}$ & 8 & 8 & - & - & - & - \\
$\begin{array}{c}\text { Cirrhotic ascites } \\
\text { (not complicated) }\end{array}$ & 178 & 172 & 5 & 1 & - & - \\
\hline
\end{tabular}

${ }^{*}$ Culture-negative neutrocytic ascites.

Table 5. Sensitivity, specificity, positive and negative predictive value, accuracy likelihood ratio for a positive and negative test of the reagent strip test to diagnose SBP considering a positive test $\geq 1, \geq 2$ or $\geq 3$ in the Colorimetric scale

\begin{tabular}{cccc}
\hline Variable & RS $\geq$ “2” (\%) & RS $\geq$ “3” (\%) & RS $\geq$ “4” (\%) \\
\hline S & 86 & 71 & 64 \\
Sp & 96 & 99 & 100 \\
PPV & 60 & 91 & 100 \\
NPV & 99 & 98 & 97 \\
Ac & 95 & 97 & 98 \\
LR+ & $0.86 /(1-0,96)$ & $0.71 /(1-0.99)$ & $0.64 /(1-1)$ \\
LR- & $(1-0.86) / 0.96$ & $(1-0.71) / 0.99$ & $(1-0.64) / 1$ \\
\hline
\end{tabular}

$\mathrm{RS}=$ reagent strip; $\mathrm{S}=$ sensibility; $\mathrm{Sp}=$ specificity; $\mathrm{PPV}=$ positive predictive value; $\mathrm{NPV}=$ negative predictive value; $A c=$ accuracy; $L R+=$ likelihood ratio for positive test; LR-=likelihood ratio for negative test.
Table 6. Area under the curve considering as test result variable the reagent strip

\begin{tabular}{llccc}
\hline \multirow{2}{*}{ AUC } & $\mathbf{p}$ & SD & \multicolumn{2}{c}{ Asymptotic 95\% confidence interval } \\
\cline { 3 - 5 } & & & Lowerbound & Upperbound \\
\hline .923 & $<0.001$ & .056 & .813 & 1.032 \\
\hline
\end{tabular}

AUC=area under the curve; $\mathrm{SD}=$ standard deviation.

Table 7. Coordinates of the ROC curve considering as test result variable the reagent strip

\begin{tabular}{ccc}
\hline Positive if equal to & Sensitivity & $\mathbf{1 - \text { Specificity }}$ \\
\hline 0 & 1.000 & 1.000 \\
1 & .857 & .043 \\
2 & .714 & .005 \\
3 & .643 & .000 \\
4 & .214 & .000 \\
\hline
\end{tabular}

Figure 1. Patient's distribution according to the reagent strip test results and PMN counts.

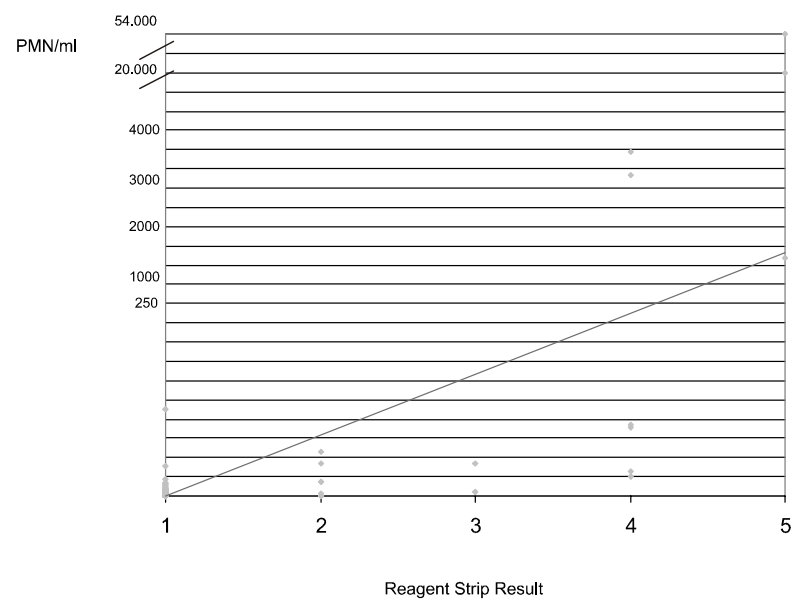

Figure 2. ROC Curve elaborated to define the cut-off of the reagent strip for considering a positive result.

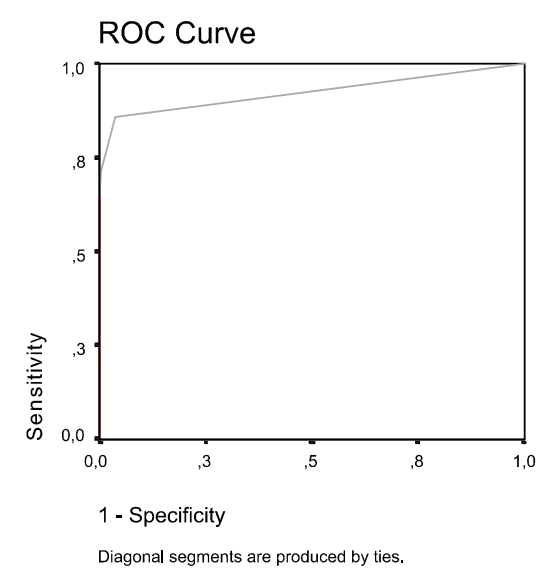


both false negative results yielded organisms (Streptococcus sp.and Candida sp.) the results of the reagent strips could perhaps be explained by lack of esterase activation in those ascitic fluid samples. Conversely, if we had considered as positive a reagent strip result of 3 or more, we would have misclassified 4 patients and overlooked the SBP diagnosis with serious consequences.

The incidence of SBP in this sample was $11 \%$ and is similar to other Brazilian studies [7,21,22]. The low incidence of such infection could be explained by the fact that most of the ascitic fluid samples were collected in the outpatient setting and is in accordance with the incidence reported in the literature for this population $[7,22]$.

The reagent strip has not only been used to rule out urinary tract infections for a long time with similar performance of detecting this kind of infection [23], but also to help diagnose patients with other biological fluid infections such as meningitis and empyema [16-18]. The use of those strips in ascitic fluid has had good results in the diagnosis of peritonitis in patients on peritoneal dialysis [24,25].

Castellote et al. studied 228 samples of ascitic fluid and diagnosed 52 episodes of SBP. The sensitivity and specificity of the reagent strip (Aution sticks ${ }^{\circledR}$; A. Menarini Diagnostics, Firenze, Italy) were $96 \%$ and $89 \%$, respectively [26]. Other studies have been carried out in order to confirm the performance of different reagent strips in SBP diagnosis in inpatient and outpatient settings reaching similar results [26-33].

Recently, Sapey et al. showed that different brands can achieve different accuracy in the diagnosis of SBP [33]. The authors compare two different reagent strips (Multistix SG x Nephur-Test) finding that the Nephur-Test seems to out-perform Multistix SG. Another study conducted by the same author that took place in two centers (Europe x USA) has not found different results with the same strip in accordance with the study center as well [29]. Hence, we consider that further studies would be necessary to determine the best reagent strip brand that should be used for the prompt SBP diagnosis.

Taking into account our results and those of these above-mentioned studies, we believe that the reagent strip could be a feasible option for a faster and cheaper diagnosis of SBP. The delay of SBP diagnosis is still frequent, mostly in hospitals with limited laboratory facilities, bringing serious consequences for life expectancy in cirrhotic patients. Thus, we should find a low-cost method that could be used anywhere, and whose results could be readily available for a fast diagnosis of this infection. The good accuracy of this method along with its simplicity and the fact that it takes only 60 seconds to complete, makes it a promising diagnostic tool that could save lives by prompting early diagnosis and treatment of this lifethreatening condition.

\section{References}

1. Almdal T.P., Skinhoj P. Spontaneous bacterial peritonitis in cirrhosis: incidence, diagnosis and prognosis. Scand J Gastroentetol 1987;22:295-300

2. Hurwich D.B., Lindor K.D., Hay J.E., et al. Prevalence of peritonitis and the ascitic fluid protein concentration among chronic liver disease patients. Am J Gastroenterol 1993;88:1254-7.

3. Jeffries M.A., Stern M.A., Gunaratnam N.T., Fontana R.J. Unsuspected infection is infrequent in asymptomatic outpatients with refractory ascites undergoing therapeutic paracentesis. Am J Gastroenterol 1999;94:2972-6.

4. Rimola A., Garcia-Tsao G., Navasa M., et al. Diagnosis, treatment and prophylaxis of spontaneous bacterial peritonitis: a consensus document. J Hepatol 2000;32:142-53.

5. Thuluvath P.J., Morss S., Thompson R. Spontaneous bacterial peritonitis - In-hospital mortality, predictors of survival, and health care costs from 1988 to 1998. Am J Gastroenterol 2001;96:1232-6.

6. Fernández J., Navasa M., Gómez J., et al. Bacterial infections in cirrhosis: epidemiological changes with invasive procedures and norfloxacin prophylaxis. Hepatology 2002;35:140-8.

7. Sette Jr. H., Mies S., Barros M.F.A., et al. Peritonite bacteriana espontânea. Rev Paul Med 1986;104:292-7.

8. Mattos A. Peritonite bacteriana espontânea e suas variantes; epidemiologia diagnóstico e história natural. GED 1994;13:97-105.

9. Guarner C., Soriano G. Spontaneous bacterial peritonitis. Seminars in Liver diseases 1997;17:203-17.

10. Moore K.P., Wong F., Gines P., et al. The management of ascites in cirrhosis: report on the consensus conference of internacional ascites club. Hepatology 2003;38:258-66.

11. Runyon B.A. Early events in spontaneous bacterial peritonitis. Gut 2004;63:782-4.

12. Guarner C., Runyon B.A. Spontaneous bacterial peritonitis: pathogenesis, diagnosis and treatment. The Gastroenterologist 1995;3:311-28.

13. Garcia-Tsao G., Conn H.O., Lerner E. The diagnosis of bacterial peritonitis: comparison of $\mathrm{pH}$, lactate concentration and leukocyte count. Hepatology 1985;5:91-6.

14. Yang C.Y., Liaw Y.F., Chu C.M., Sheen I.S. White count, $\mathrm{pH}$ and lactate in ascites in the diagnosis of spontaneous bacterial peritonitis. Hepatology 1985;5:85-9.

15. Runyon B.A., Antillon M.R. Ascitic fluid $\mathrm{pH}$ and lactate: insensitive and nonespecific tests in detecting ascitic fluid infection. Hepatology 1991;13:929-35.

16. Moosa A.A., Quortum H.A., Ibrahim M.D. Rapid diagnosis of bacterial meningitis with reagent strips. Lancet 1995;345:1290-1.

17. Azoulay E., Fartouk M., Galliot R., et al. Rapid diagnosis of infectious pleural effusions by use of reagent strips. Clin Infect Dis 2000;31:914-9.

18. Castellote J., Lopez C., Gornals J., et al. Use of reagent strips for the rapid diagnosis of spontaneous bacterial empyema. J Clin Gastroenterol 2005;39:278-81.

19. Garcia-Tsao G., Grace N.D., Groszmann R.J., et al. Short-term effects of propranolol on portal venous pressure. Hepatology 1986;6:101-6.

20. Evans L.T., Kim W.R., Poterucha J.J., Kamath P.S. Spontaneous bacterial peritonitis in asymptomatic outpatients with cirrhotic ascites. Hepatology 2003;37:897-901.

21. Figueiredo F.A.F., Coelho H.S.M., Soares J.A.S. Peritonite bacteriana espontânea na cirrose hepática: prevalência, fatores preditivos e prognóstico. Rev Ass Med Brasil 1999;45:128-36.

22. Coral G., Mattos A.A., Damo D.F., Viégas A.C. Prevalência e prognóstico da peritonite bacteriana espontânea. Experiência em pacientes internados em um hospital geral de Porto Alegre, RS, Brasil (1991-2000). Arq. Gastroenterol 2002;39:158-62.

23. Devillé W.L., Yzermans J.C., van Duijn N.P., et al. The urine dipstick test useful to rule out infections. A meta analysis of the accuracy. BMC Urology 2004; Jun 2;4:4. 
24. Sam R., Sahani M., Ulozas E., et al. Utility of a peritoneal dialysis leokocyte strip in diagnosis of peritonitis. Aritf Organs 2002;26:546-48.

25. Farmer C.K., Hobbs H., Mann S., et al. Leukocyte esterase reagent strips for early detection of peritonitis in patients on peritoneal dyalisis. Perit Dial Int 2000;20:237-9.

26. Castellote J., López C., Gornals J., et al. Rapid diagnosis of spontaneous bacterial peritonitis by use of reagent strips. Hepatology 2003,37:893-6.

27. Vanbiervliet G., Rakotoarisoa C., Filippi J., et al. Diagnostic accuracy of a rapid urine-screening test (Multistix8SG) in cirrhotic patients with spontaneous bacterial peritonitis. Eur J Gastroenterol Hepatol 2002,14:1257-60.

28. Thèvenot T., Cadranel J.F., Nguyen-Khac E., et al. Diagnosis of spontaneous bacterial peritonitis in cirrhotic patients by use of two reagent strips. Eur J Gastroenterol Hepatol 2004;16:579-83.
29. Sapey T., Mena E., Fort E., et al. Rapid diagnosis of spontaneous bacterial peritonitis with leukocyte esterase reagent strips in a European and in an American center. J Gastroentetol Hepatol 2005;20:187-92.

30. Kim do Y., Kim J.H., Chon C.Y., et al. Usefulness of urine strip test in the rapid diagnosis of spontaneous bacterial peritonitis. Liver Int 2005;25:1197-201.

31. Wisniewski B., Rautou P.E., Al Sirafi Y., et al.Diagnosis of spontaneous ascites infection in patient with cirrhosis reagent strips. Presse Med 2005;34:997-1000.

32. Sarwar S., Alam A., Izhar M., et al. Bedside diagnosis of spontaneous peritonitis using reagent strips. J Coll Physicians Surg Pak 2005; $15: 418-21$.

33. Sapey T., Kabissa D., Fort E., et al. Instant diagnosis of spontaneous bacterial peritonitis using leukocyte esterase reagent strips: Nephur-Test vs. Multistix SG. Liver Int 2005;25:343-8. 